proteins associated with telomeric repeats. Transcription of telomeric repeats is a conserved feature revealed in many species, and the mechanisms of the telomere homeostasis are closely related to regulation of the telomeric repeat transcription. The telomeres of Drosophila are maintained in the absence of telomerase by the transpositions of the specialized telomeric retrotransposons. Using the Drosophila model, we study the relationship between the telomeric chromatin state and telomeric repeat expression in the female germline. In this study, we have examined the changes in the telomeric chromatin structure and nuclear telomere positioning induced by massive overexpression of telomeric repeats using RNA/DNA fluorescence in situ hybridization, immunostaining and chromatin immunoprecipitation methods. Heterochromatin protein 1 (HP1) and H3K9me3 are important components of telomeric chromatin in different species; however, the mechanisms underlying their deposition at the telomere are not clear. Disruption of the PIWI-interacting RNA pathway causes overexpression of telomeric repeats, a loss of heterochromatic components, and translocation of telomeres from the periphery to the nuclear interior. However, piRNAs are not required either for assembly of the protective telomere capping complex or for telomere clustering in the germline. We have revealed that piRNAs provide a germline-specific mechanism for HP1, Rhino, a germlinespecific HP1 homolog, and H3K9me3 deposition at telomeric retrotransposon arrays, thus ensuring telomere silencing at the transcription level during gametogenesis. It has also been found that telomeric transcript levels are regulated by the factors of co-transcriptional RNA surveillance, such as the deadenylase Ccr4-Not complex and nuclear RNA-binding protein Ars2. Noteworthy, the mechanisms for nuclear metabolism of telomeric transcripts are highly conserved. Indeed, Ars2 suppresses transcription of telomeric repeats both in human cells and in Drosophila. Depletion of the factors of co-transcriptional RNA metabolism results in the accumulation of Drosophila telomeric transcripts at transcription sites without the loss of heterochromatin marks. In this case, telomeres remain localized to the nuclear periphery. Taken together, these data indicate that transcription and chromatin states of telomeres rather than accumulation of chromatin-associated telomeric transcripts determine a peripheral localization of telomeres in the Drosophila germline.

Russian Foundation for Basic Research (19-04-00254)

doi: http://dx.doi.org/10.7124/bc.0009E6

\section{K-2. Role of ERK 1/2 pathway in polyploidization of hepatocytes in cholestatic liver}

S. Kiparoidze, E. Bakuradze, I. Modebadze, D. Dzidziguri, K. Agagulian

Department of Biology, Iv. Javakhishvili Tbilisi

State University, Tbilisi, Georgia

salo_kipa@yahoo.com

In response to resection, the importance of cell proliferation, polyploidization and hypertrophy for liver regeneration and their contribution to rapid recovery of functions is well studied. However, relatively little information exists about liver regeneration 
in various pathologies where all three mechanisms mentioned above do not happen simultaneously. For example, it's established that: during alimentary dyslipidemia, mechanism of regeneration depends on the duration of hepatogenic diet uses and quality of the damage. In cholestatic liver of rat, at initial stage regeneration is only occurs by polyploidization without increasing of cells quantity. In response to trauma, increase of ploidy is also described in different mammalians tissues (heart, liver, and cornea). Based on the above, in some pathologies liver regeneration is achieved by the quantitative increase of ploidy cells, however, it is not known which signaling pathways activation/inactivation provide their appearance in each particular case. Our previous results show that inhibition of HGF receptor (C-Met) doesn't cause the changes of polyploidization of cholestatic liver. ERK $1 / 2$ is involved in HGF activated pathway where MEK 1 and MEK 2 are the key molecules, which can be also activated by avoiding of main receptor - C-Met. The aim of the research is the determination of role of ERK 1/2 signaling pathways in the process of hepatocytes polyplodization in cholestatic liver model. Materials and methods. Experiments were carried out on adult white rats $(130-150 \mathrm{~g})$. Model of cholestatic liver with common bile duct ligation was used. Animals were divided into three groups: I-control intact animals, II -cholestatic animals (2nd day), IIIcholestatic animals with MEK 1/2 (PD98059) inhibitor injection $(10 \mathrm{mg} / \mathrm{kg})$. Nuclear DNA content was detected by using of computer software Image J 1.36b. Determination of colchicine mitotic index was used for assess- ment of proliferative activity. Results. Hepatocytes mitotic activity significantly increases on the 2 nd day from common bile duct ligation in the II group. In addition, the number of diploid (2c) hepatocytes decreases and the polyploid $(2 \mathrm{c} \times 2,4 \mathrm{c}, 4 \mathrm{c} \times 2,8 \mathrm{c})$ cells are increased. In the III group of animals, the number of tetrapoloid cells (4c) has tendency to decreased, while octaploid (8c) and binuclear octoploid $(4 \mathrm{c} \times 2)$ cells are not present, which is evidence for suppression of polyploidization. With regard to proliferative activity, there is no difference between the animals from II and III group. Conclusions. Inhibition of formation of highploidy (octaploid) cells by blocking of MEK $1 / 2$ proteins indicates that ERK $1 / 2$ signaling pathway is one of the necessary conditions for polyploidization of hepatocytes.

doi: http://dx.doi.org/10.7124/bc.0009E7

\section{K-3. Molecular evolution of the histone variant $\mathrm{H} 2 \mathrm{~A} . \mathrm{Z}$}

Saho Kitagawa $^{1}$, Masayuki Kusakabe ${ }^{2}$, Hiroyuki Oku${ }^{1}$, Daisuke Takahashi ${ }^{1}$, Takumi Narimiya ${ }^{1}$, Yu Nakabayashi ${ }^{3}$, Masayuki Seki $^{3}$, Masahiko Harata ${ }^{1}$

${ }^{1}$ Tohoku University, Japan; ${ }^{2}$ Kobe University, Japan; ${ }^{3}$ Tohoku Medical and Pharmaceutical University, Japan

mikan3kan0905@gmail.com

Histone variants contribute to the organization of chromatin structures and regulation of genome functions through their deposition into nucleosome. Histone variant H2A.Z, one of the H2A variants, is evolutionarily con- 